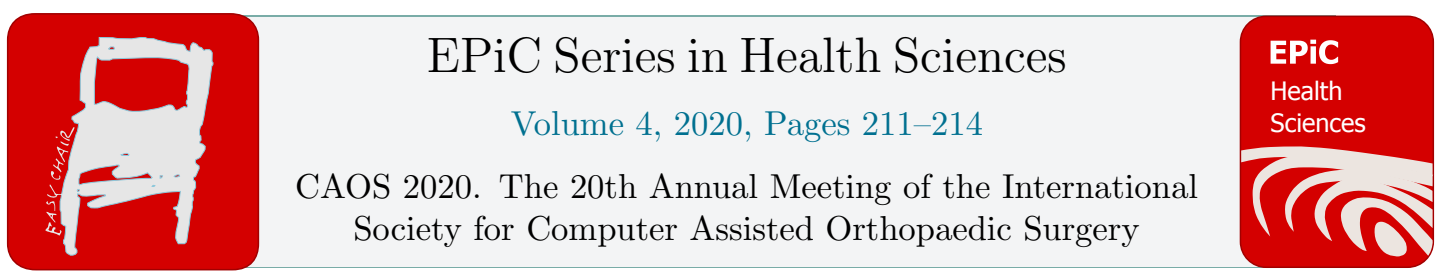

\title{
Towards markerless 3D pose estimation for Computer Assisted Orthopaedic Surgery: a comparison study of depth cameras
}

\author{
Jérôme Ogor ${ }^{1}$, Guillaume Dardenne ${ }^{1}$, Salahhedine Sta ${ }^{2}$, Julien Bert ${ }^{1}$, Hoël \\ Letissier $^{1}$, Eric Stindel ${ }^{1}$ and Chafiaa Hamitouche ${ }^{2}$ \\ ${ }^{1}$ LaTIM UMR1101 INSERM \\ ${ }^{2}$ Institut Mines-Télécom Atlantique Bretagne-Pays de la Loire \\ jerome.ogor@inserm.fr
}

\begin{abstract}
This abstract addresses the problem of localizing surgical instruments during orthopaedic surgeries. Compared to usual approaches based on surgical navigation with markers, we propose here a novel method that estimates the 6-DoF pose of surgical instruments without specific markers using a depth camera. The goal of this paper is to compare, on real data, the registration precision of an algorithm called Point Pair Features (PPF) according to consumer depth cameras available on the market. Experimental validation using sawbones has been conducted and 8 cameras have been tested in realistic clinical environment. The Kinect Azure reports the best precision with a registration error of $1.13 \mathrm{~mm} \pm 1.00 \mathrm{~mm}$.
\end{abstract}

\section{Introduction}

Knee arthritis causes cartilage between the bone joints to wear away and often leads to patient's joint pain. Total Knee Arthroplasty (TKA) is a common response to this disease and [1] forecasts a growth by $673 \%$ up to 3.48 million TKA procedures in the USA by 2030 . Yet, around $24 \%$ of TKA patients declares being unsatisfied with the postoperative results [2]. Besides, surgeons are traditionally guided by navigation systems during the operation. However, these solutions require both expensive sensors and additional time and complexity in the surgical workflow mainly due to the markers attached on patient bones. Therefore, we propose to evaluate the precision of an algorithm called Point Pair Features (PPF) [3] which could be used to estimate intraoperatively the 3D pose of orthopaedic cut guides. The goal is to compare the precision of different general public depth cameras according to this algorithm. 


\section{Methods}

The PPF algorithm was tested on ex-vivo realistic data: we placed the surgical cut guide on a sawbone at $50 \mathrm{~cm}$ from the depth sensor in an operating room with scialytic lighting conditions and measured this error for a dataset of 100 real scenes for each of the 8 evaluated depth camera. Thus we evaluated the 3D pose estimation PPF algorithm on around 800 real data captured by the cameras described in the table 1. The calculated registration error corresponds to the distance between points belonging to the cut guide in the scene and the registered model mesh computed by the PPF algorithm. This error defines a distance map between the registered model and the scene.

\begin{tabular}{|c|c|c|c|c|}
\hline Camera & Technology & $\begin{array}{l}\text { Range } \\
\text { (m) }\end{array}$ & $\begin{array}{l}\text { Depth } \\
\text { Resolution } \\
+ \text { FPS }\end{array}$ & $\begin{array}{l}\text { Field of } \\
\text { View }\end{array}$ \\
\hline Intel RealSense D415 & $\begin{array}{l}\text { Active Stereo } \\
\text { (rolling shutter) }\end{array}$ & $0.16-10$ & $\begin{array}{c}1280 \times 720 \\
@ 30 \mathrm{FPS}\end{array}$ & $\begin{array}{l}63^{\circ} \text { Hor } \\
40^{\circ} \text { Ver }\end{array}$ \\
\hline Intel RealSense D435 & $\begin{array}{l}\text { Active Stereo } \\
\text { (global shutter) }\end{array}$ & $0.11-10$ & $\begin{array}{l}848 \times 480 \\
@ 30 \mathrm{FPS}\end{array}$ & $\begin{array}{l}85^{\circ} \text { Hor } \\
58^{\circ} \text { Ver }\end{array}$ \\
\hline Orbbec Astra S & Structured Light & $0.4-2$ & $\begin{array}{l}\text { 640x480 } \\
\text { @ } 30 \mathrm{FPS}\end{array}$ & $\begin{array}{l}60^{\circ} \text { Hor } \\
50^{\circ} \text { Ver }\end{array}$ \\
\hline Orbbec Astra Embedded S & Structured Light & $0.25-1.5$ & $\begin{array}{l}1280 \times 800 \\
@ 30 \mathrm{FPS}\end{array}$ & $\begin{array}{l}68^{\circ} \text { Hor } \\
45^{\circ} \text { Ver }\end{array}$ \\
\hline Occipital Structure Sensor & Structured Light & $0.4-3.5$ & $\begin{array}{l}\text { 640x480 } \\
\text { @ } 30 \mathrm{FPS}\end{array}$ & $\begin{array}{l}58^{\circ} \text { Hor } \\
45^{\circ} \text { Ver }\end{array}$ \\
\hline Occipital Structure Core & $\begin{array}{l}\text { Active Stereo } \\
\text { (global shutter) }\end{array}$ & $0.3-10$ & $\begin{array}{l}\text { 1280x960 } \\
\text { @ } 54 \text { FPS }\end{array}$ & $\begin{array}{l}59^{\circ} \text { Hor } \\
46^{\circ} \text { Ver }\end{array}$ \\
\hline Microsoft Kinect V2 & Time of Flight & $0.5-4.5$ & $\begin{array}{l}512 \times 424 \\
\text { (a) } 30 \mathrm{FPS}\end{array}$ & $\begin{array}{l}70^{\circ} \text { Hor } \\
60^{\circ} \text { Ver }\end{array}$ \\
\hline Microsoft Kinect Azure & Time of Flight & $0.25-5.46$ & $\begin{array}{c}1024 \times 1024 \\
\text { @ } 15 \mathrm{FPS}\end{array}$ & $\begin{array}{l}120^{\circ} \text { Hor } \\
120^{\circ} \text { Ver }\end{array}$ \\
\hline
\end{tabular}

Table 1: List of evaluated depth cameras and hardware specifications

\section{Results}

To begin with, both D435 and D415 provide respectively a mean registration error of $2.83 \mathrm{~mm} \pm$ $1.80 \mathrm{~mm}$ and $2.67 \mathrm{~mm} \pm 1.52 \mathrm{~mm}$ and many false positives estimated pose of the cut guide mainly due to the poor raw depth accuracy in presence of scialytic illuminations. KinectV2 performs also poorly because of the sensor low resolution which generates too few points to localize the surgical instrument in the scene for PPF algorithm. Then Astra S, Embedded S as well as Structure Sensor and Core give decent similar results (median around 1.30mm). Finally, the Microsoft Kinect Azure shows the best results with a mean registration error of millimeter order $(1.13 \mathrm{~mm} \pm 1.00 \mathrm{~mm})$. Results are provided in the Figure 1. 


\begin{tabular}{|c|c|c|c|c|c|}
\hline Camera & $\begin{array}{c}\text { Mean error } \\
(\mathrm{mm})\end{array}$ & $\begin{array}{c}\text { Standard deviation } \\
(\mathrm{mm})\end{array}$ & $\begin{array}{c}\text { Median } \\
(\mathrm{mm})\end{array}$ & $\begin{array}{c}\text { Minimum } \\
(\mathrm{mm})\end{array}$ & $\begin{array}{c}\text { Maximum } \\
(\mathrm{mm})\end{array}$ \\
\hline Intel RealSense D435 & 2.83 & 3.08 & 1.80 & 0.000864 & 21.4 \\
\hline Intel RealSense D415 & 2.67 & 3.79 & 1.52 & 0.000580 & 30.4 \\
\hline Microsoft Kinect V2 & 3.73 & 4.50 & 2.13 & 0.000825 & 42.3 \\
\hline Orbbec Astra S & 1.26 & 0.791 & 1.29 & 0.00000724 & 5.48 \\
\hline Occipital Structure Sensor & 1.30 & 0.75 & 1.35 & 0.000882 & 5.11 \\
\hline Occipital Structure Core & 1.76 & 2.05 & 1.36 & 0.00000603 & 40.3 \\
\hline Microsoft Kinect Azure & $\mathbf{1 . 1 3}$ & $\mathbf{1 . 0 0}$ & $\mathbf{0 . 8 5}$ & $\mathbf{0 . 0 0 0 1 5 5}$ & $\mathbf{8 . 3 1}$ \\
\hline Orbbec Astra Embedded S & 1.29 & 0.76 & 1.39 & 0.000500 & 31.7 \\
\hline
\end{tabular}

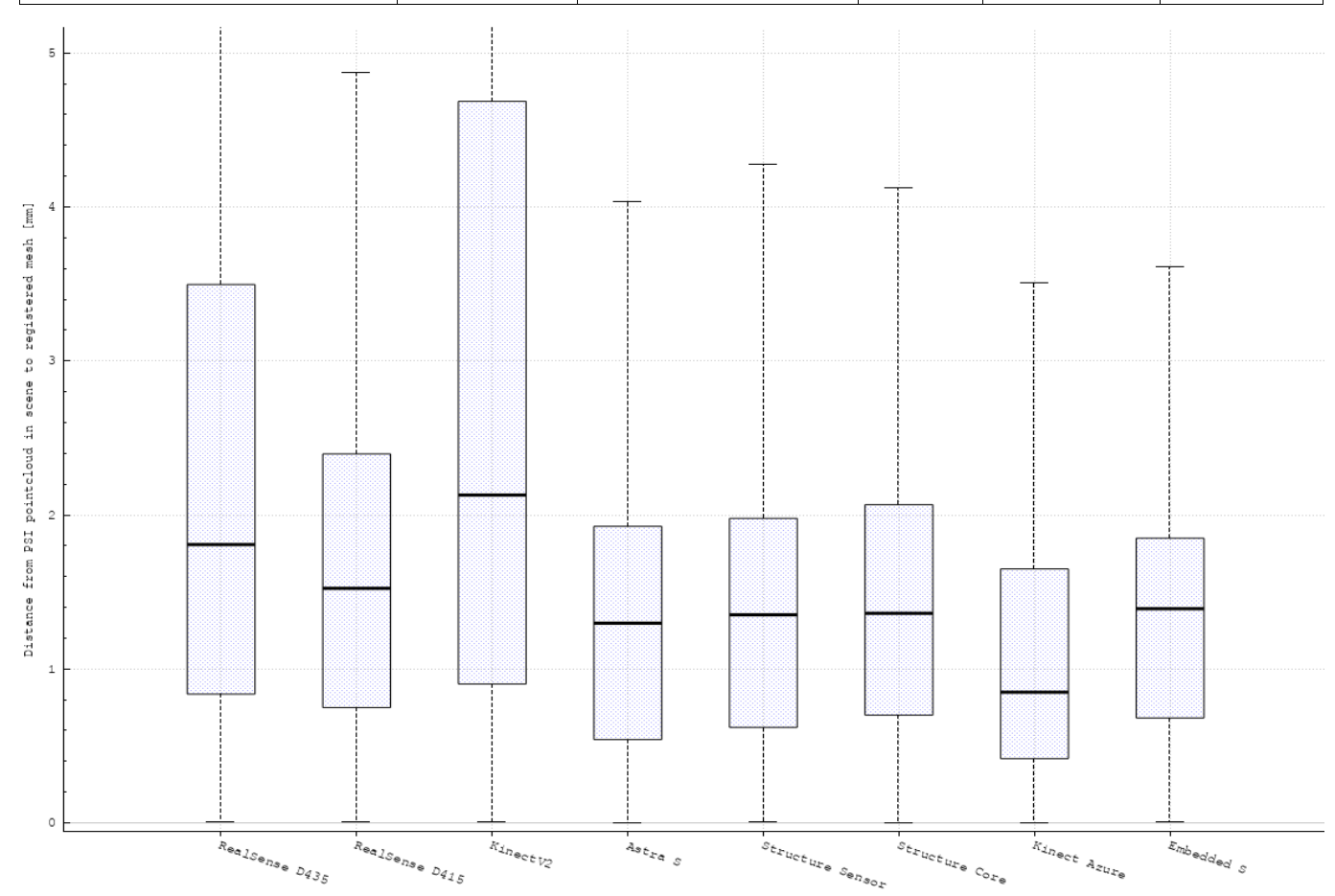

Figure 1: Statistical precision of compared depth cameras for 3D pose estimation

\section{Conclusion}

This study intended to compare low cost depth cameras precision for the markerless 3D localization task in the computer assisted orthopaedic surgery context. Not only such approach could reduce both intervention complexity and time associated to existing surgical navigation systems but also proved to be relevant in terms of precision. Indeed, the Kinect Azure shows a distance map mean registration error of $1.13 \mathrm{~mm}$, which could be suitable for TKA. Moreover, the results are very encouraging compared to the state of the art: [4] is similar to our work and intends to register intraoperatively bone surface with depth camera with a precision of $6.18 \mathrm{~mm}$. [5] also reports an 
higher average registration error of $11.46 \mathrm{~mm}$ on real data for similar task. However, the 3D pose estimation algorithm precision could be further compared to traditional orthopaedic navigation with translation and angular error metrics and this proof of concept could also be pushed to even more realistic environment with cadaver soft tissues.

\section{Acknowledgment}

This work benefited from State aid managed by the National Research Agency under the future investment program bearing the reference ANR-17-RHUS-0005.

\section{References}

[1] Kurtz S, Ong K, Lau E, Mowat F, Halpern M. Projections of primary and revision hip and knee arthroplasty in the United States from 2005 to 2030. J Bone Joint Surg Am. 2007

Apr;89(4):780-5.

[2] Varacallo M, Chakravarty R, Denehy K, Star A. Joint perception and patient perceived satisfaction after total hip and knee arthroplasty in the American population. J Orthop. 2018 Jun;15(2):495-499.

[3] J. Vidal, C.-Y. Lin, and R. Marti, "6D pose estimation using an improved method based on point pair features," in2018 4th International Conference on Control, Automation and Robotics (ICCAR), (Auckland), pp. 405-409,IEEE, Apr. 2018.

[4] Rodrigues, P., Antunes, M., Raposo, C., Marques, P., Fonseca, F., Barreto, J. (2019). Deep segmentation leverages geometric pose estimation in computer-aided total knee arthroplasty.

Healthcare Technology Letters. 10.1049/ht1.2019.0078.

[5] Jonas Hajek, Mathias Unberath, Javad Foutouhi, Bastian Bier, Sing Chun Lee, Greg Osgood, Andreas Maier, Mehran Armand and Nassir Navab. (2018). Closing the Calibration Loop: An Inside-Out-Tracking Paradigm for Augmented Reality in Orthopedic Surgery. Medical Image Computing and Computer Assisted Intervention 2018 (4): 299-306. 